\title{
POPULATION-SIZE-DEPENDENT BRANCHING PROCESSES ${ }^{1}$
}

\author{
PETER JAGERS \\ Chalmers University of Technology \\ and \\ Gothenburg University \\ S-412 96 Gothenburg, Sweden
}

(Received August, 1996; Revised October, 1996)

\begin{abstract}
In a recent paper [7] a coupling method was used to show that if population size, or more generally population history, influence upon individual reproduction in growing, branching-style populations disappears after some random time, then the classical Malthusian properties of exponential growth and stabilization of composition persist. While this seems self-evident, as stated, it is interesting that it leads to neat criteria via a direct Borel-Cantelli argument: If $m(n)$ is the expected number of children of an individual in an $n$-size population and $m(n) \geq m>1$, then essentially

$$
\sum_{n=1}^{\infty}\{m(n)-m\}<\infty
$$

suffices to guarantee Malthusian behavior with the same parameter as a limiting independent-individual process with expected offspring number $m$. (For simplicity the criterion is stated for the single-type case here.)

However, this is not as strong as the results known for the special cases of Galton-Watson processes [10], Markov branching [13], and a binary splitting tumor model [2], which all require only something like

$$
\sum_{n=1}^{\infty}\{m(n)=m\} / n<\infty
$$

This note studies such latter criteria more generally. It is dedicated to the memory of Roland L. Dobrushin.
\end{abstract}

Key words: Branching Processes, Population Dynamics, Cell Kinetics.

AMS (MOS) subject classifications: $60 \mathrm{~J} 80,60 \mathrm{~F} 25,92 \mathrm{~A} 15$.

\section{Introduction}

Independence between individuals, once born into the population, lies at the very heart of branching processes. Without it, virtually no traditional methods work. But the independence assumption is no crude approximation for mathematical convenience: population dynamics is driven by individual reproduction. Conceptually the latter comes before the former, and independence emerges as a natural idealization of this precedence.

But to what extent does typical branching process behavior persist in less ideal models, where

\footnotetext{
${ }^{1}$ Support from The Swedish Natural Sciences Research Council is acknowledged.
} 
there is interaction between individuals, or between the population as a whole and individual behavior? We study an aspect of this in the supercritical case, viz. the persistence of Malthusian exponential growth, and the ensuing Eulerian stabilization of composition.

In a seminal paper, Klebaner [10] managed to include population size dependence into simple Galton-Watson processes: Presume that the expected reproduction by an individual belonging to a generation of size $n$ is $m(n) \downarrow m>1$, then, besides some technical side conditions,

$$
\sum_{n}\{m(n)-m\} / n<\infty
$$

suffices to guarantee that the population in generation $k, z_{k}$, grows as $m^{k}$.

It is interesting that Gyllenberg and Webb [2] have established essentially the same criterion for balanced exponential growth of a Bell-Anderson type tumor model, where newborn cells can move into (and out of) quiescence at a tumor-size dependent rate. We shall discuss an agedependent variation of this: When a cell is born, assume that it enters the so-called $G_{0}$ state of eternal quiescence with a probability $1-p(n)$, where $n$ is the population (i.e., tumor) size at the moment of birth. Otherwise, with probability $p(n)$ the cell embarks upon the cell cycle, which is assumed to have i.i.d. lengths with an arbitrary but continuous distribution function, and ends by cell division. It is assumed that the growth rate decreases with tumor size, so that $1 \geq$ $p(n) \downarrow p>1 / 2$, as $n \rightarrow \infty$. The Gyllenberg and Webb condition for the same balanced exponential growth as would have been the case in a tumor-size independent model, $p(n) \equiv p$, would then be

$$
\sum\{p(n)-p\} / n<\infty \text {. }
$$

As $m(n)=2 p(n)$ in these binary splitting models, it seems that Klebaner and Gyllenberg and Webb have spotted a condition which is fundamental also in a broader perspective.

In [7] population history dependence was investigated quite generally, by a coupling of individual reproductions to those of a limiting independent-reproduction branching population. For the special case of population-size-dependent single-type populations, the (necessary and sufficient) condition for success of that coupling, however, turned out to be of the form

$$
\sum_{n}\{m(n)-m\}<\infty .
$$

In this note we investigate to what extent the weaker conditions suffice more generally. A general growth condition, the first inequality of Theorem 1, is formulated to express the property that in a growth tree no node may be expected have a much bigger daughter tree than the other nodes on the same level. This seems to be the conceptual background of the $1 / n$-factor, $n$ being the number of nodes at a level.

\section{Framework}

Let us recall the setting, as given in [5]. Let

$$
I=\bigcup_{n=0}^{\infty} N^{n}, N^{0}=\{0\}, N=\{1,2, \ldots\}
$$

denote the Ulam-Harris space of all possible individuals. Think of the population as initiated by an Eve, whose name in $I$ is 0 , and the $x(k):=(x, k) \in I$ as $x$ 's $k$ th child, twins, etc. numbered in an arbitrary way. Assume that each $x \in I$ has a reproduction process $\xi_{x}$, giving $x$ 's age at her successive child-bearings and the types of the children then born, the latter being elements of a type space $(S, \varphi)$ with a countably generated $\sigma$-algebra.

Thus, there is a sequence of maps $\left(\tau_{x}(k), \sigma_{x}(k)\right), k=1,2, \ldots, 0 \leq \tau_{x}(1) \leq \tau_{x}(2) \leq \ldots \leq \infty$, $\sigma_{x}(k)$ with values in $S$ and the interpretation that $\tau_{x}(k)$ is $x$ 's age at giving birth to her $k$ th child and $\sigma_{x}(k)$ is that child's type. Of course, if $\tau_{x}(k)<\tau_{x}(k+1)=\infty$, then $x$ never has more than $k$ 
children, and the corresponding individuals in $I$ are not realized. Clearly,

$$
\xi_{x}(A \times B)=\#\left\{k \in I ; \sigma_{x}(k) \in A, \tau_{x}(k) \in B\right\} .
$$

The birth time and type of an individual $x \in I$ are denoted by $\tau_{x}$ and $\sigma_{x}$, respectively. These are inductively given from a starting type $\sigma_{0} \in S$ and $\tau_{0}=0$.

Population size can be measured by random characteristics, i.e., at time $t$ the individual $x$ is assumed to have some weight $\chi_{x}\left(\sigma_{x}, t-\tau_{x}\right)$, where, $\chi_{x}$ is a functional $\chi$ as evaluated on $x$ 's daughter process. More precisely, define $S_{x}$ to be the coordinate projection, restricting attention from the space of all lives to only the life of $x$ and all her progeny. In other words, $S_{x}$ renders $x$ the ancestor in a space, which otherwise has the same structure as the original space $\Omega$ of all possible life careers of all possible individuals. A characteristic is a measurable map $\chi: S \times \mathbb{R}_{+} \times$ $\Omega \rightarrow \mathbb{R}_{+}$, which is $D$-valued in its second coordinate, interpreted as age. Further, $\chi_{x}:=\chi \circ S_{x}$. Thus, an individual's weight, once her type and age are given is determined by her own life, and possibly her progeny's lives. We assume characteristics to be nonnegative, bounded, and vanishing for negative ages. Typical characteristics would be $1_{S \times \mathbb{R}}$, or $1_{A \times\left[0, \min \left(\lambda_{x}, a\right)\right]}$ for $A \in \varphi$. Such characteristics, and many other, are individual in the sense that they actually depend only upon $x$ 's own life, type, and age, and remain unaffected by $x$ 's progeny.

The $\chi$-counted population size at time $t$ is then defined as

$$
z_{t}^{\chi}:-\sum_{x \in I} \chi_{x}\left(\sigma_{x}, t-\tau_{x}\right)
$$

Thus, the characteristic $1_{S \times \mathbb{R}}$ yields the total population, i.e., all those born, and the other characteristic mentioned gives the number of those alive of type $A$ and not older than $a$. Though it is important to vary the characteristics, in order to catch the asymptotic composition of the population, we shall usually consider a fixed but arbitrary such entity $\chi$, and then sometimes write just $z_{t}$ for $z_{t}^{\chi}$.

In the classical case of independent reproduction, the reproduction process depends upon the past only through the individual type and the form of supercritical population growth is essentially governed by the reproduction kernel $\mu$, defined as the expected number births of children of various types and at various ages:

$$
\mu(r, d s \times d t):=E_{r}[\xi(d s \times d t)],
$$

$r$ being the type of the individual with reproduction $\xi$. The usual assumption is that the population is Malthusian and supercritical, this meaning that there is a number $\alpha>0$, the Malthusian parameter, such that the kernel $\widehat{\mu}(\alpha)$,

$$
\widehat{\mu}(r, d s ; \alpha):=\int_{0}^{\infty} e^{-\alpha t} \mu(r, d s \times d t)
$$

has Perron root one and is what Shurenkov [24] calls conservative. (This corresponds to irreducibility and $\alpha$-recurrence in the terminology of [18].) By the abstract Perron-Frobenius theorem ([24, p. 43$]$ or $[19$, p. 70$]$, there is then a $\sigma$-finite measure $\pi$ on the type space $(S, \varphi)$, and strictly positive a.e., $[\pi]$ finite measurable function $h$ on the same space, such that

$$
\begin{aligned}
\int_{S} \widehat{\mu}(r, d s ; \alpha) \pi(d r) & =\pi(d s), \\
\int_{S} h(s) \widehat{\mu}(r, d s ; \alpha) & =h(r) .
\end{aligned}
$$

Further, strong or positive $\alpha$-recurrence in the sense that $h \in L^{1}[\pi]$ is required and 


$$
0<\beta=\int_{S \times S \times R_{+}} t e^{-\alpha t} h(s) \mu(r, d s \times d t) \pi(d r)<\infty .
$$

(In population dynamics this entity might be interpreted as the long-run stable age at childbear ing.) Then, one norms to

$$
\int_{S} h d \pi=1
$$

and makes the homogeneity assumption that inf $h>0$. Then $\pi$ is finite and can (and will) also be normed to a probability measure. These are the conditions (i.e., those concerning $\mu$ ) for the general Markov renewal theorem of [24, p. 107], which in the non-lattice case lead to the expected asymptotics

$$
\mathbb{E}_{s}\left[z_{t}^{\chi}\right] \sim h(s) \mathbb{E}_{\pi}[\widehat{\chi}(\alpha)] e^{\alpha t} / \alpha \beta,
$$

as $t \rightarrow \infty$. (Here hat denotes the Laplace transform, so that

$$
\widehat{\chi}(\alpha)=\alpha \int_{0}^{\infty} e^{-\alpha t} \chi(\sigma, t) d t
$$

where $\sigma$ denotes the type, following the stable type distribution $\pi$, as indicated by the suffix of the expectation, $\mathbb{E}_{\pi}:=\int_{S} \mathbb{E}_{s} \pi(d s)$, and the necessary direct-Riemann-type regularity assumptions on the expected characteristic are tacitly presumed.)

The fundamental finding of the theory of general, supercritical branching processes is that under very broad conditions, this leads on to a similar behavior of the process itself, in $L^{1}, L^{2}$, in probability, or a.s. for $\pi$-almost-all $\mathbb{P}_{s}$,

$$
z_{t}^{\chi} \sim w \mathbb{E}_{\pi}[\widehat{\chi}(\alpha)] e^{\alpha t} / \alpha \beta
$$

where $w$ is a random variable, vanishing precisely if the process dies out and with mean value $\mathbb{E}_{s}[w]=h(s)$. The fact that this holds for many different $\chi$ 's is what is referred to as balanced exponential growth in certain branches of biology and as asynchronous exponential growth in (deterministic) mathematical population dynamics. Here we shall bypass the technical conditions for such growth (cf. [5]), and instead assume directly that the limiting, classical independent-individual, branching population exhibits balanced exponential growth. Somewhat inadvertently, we shall summarize this by referring to the (latter) population as (non-lattice) Malthusian, adding the particular character of the asymptotics (a.s., in probability, or in the mean) whenever necessary. The populations under study will be referred to as actual or history-dependent. It will be assumed that they are always finite (at finite times). If they exhibit the exponential asymptotics of the formula above they will also be called Malthusian and the entities $\alpha, h$, and $\pi$ will be referred to as their Malthusian parameter, fitness function and stable type distribution, respectively, as for populations with independently acting individuals.

By the assumption that history-dependent populations remain finite at finite times, individuals can be numbered as they appear, starting from the ancestor $X_{1}$ and continuing $X_{2}, X_{3}, \ldots$, simultaneously born individuals - if any - so numbered that progeny can never precede their ancestors. A filtration $\left\{\mathscr{F}_{n}\right\}$ of $\sigma$-algebras is defined by letting $\mathscr{F}_{n}$ be generated by the complete lives of all individuals $X_{1}, X_{2}, \ldots, X_{n}$. Define $\mathcal{G}_{x}$ by

$$
A \in \mathcal{G}_{x} \Leftrightarrow A \cap\left\{X_{n}=x\right\} \in \mathcal{F}_{n-1},
$$

for all $n$. If no individuals are ever born together, this means that $\oint_{x}$ is the $\sigma$-algebra generated by the complete lives of all individuals born before $x$. The reason for this seemingly complicated, stopping line type definition is to cover possible simultaneous births. The time $\tau_{x}$ when $x \in I$ is born is measurable with respect to $\mathcal{G}_{x}$, as is $x$ 's type $\sigma_{x}$ (with a suitable convention for never real- 
ized individuals).

We consider the situation where individual reproduction tends to exceed what it would have been in an imaginary infinite population, "tends to exceed" being interpreted as "stochastically larger than". In other words, if $x \in I, \tau_{x}<\infty$, and $\eta$ is a generic imaginary reproduction point process, then the basic assumption is that for all $u$ and sets $A, B$,

$$
\mathbb{P}\left(\xi_{x}(A \times B)>u \mid \mathfrak{g}_{x}\right) \geq \mathbb{P}_{\sigma_{x}}(\eta(A \times B)>u),
$$

where the right-hand-side $\mathbb{P}$-suffix indicates the dependence upon type of reproduction a general branching process. Let $\mathbb{P}^{x}$ denote a regular version of the conditional distribution, given $\mathcal{G}_{x}$, supposed to exist. By Strassen's theorem [16, p. 129] we may assume that for each $x \in I$, there is a pair of reproduction point processes $\left(\xi_{x}, \eta_{x}\right)$ such that $\xi_{x}$ is $x$ 's original reproduction, whereas $\eta_{x}$ is the imaginary reproduction, "if the population were already infinite," and $\mathbb{P}^{x}\left(\xi_{x} \geq \eta_{x}\right)=1$ is the obvious partial order of measures. By an Ionesco-Tulcea construction (cf. [5]) we may assume that the pairs $\left(\xi_{x}, \eta_{x}\right)$ are defined on a joint population probability space with the proper conditional distributions and still satisfying $\xi_{x} \geq \eta_{x}$ a.s.

\section{Results}

The ordering $\xi_{x} \geq \eta_{x}$ means that the imaginary reproduction can be thought of as arising from the actual, population-history-dependent reproduction through abortion of some children. Write $\delta_{x}$ to indicate abortion or not of $x$, so that

$$
\eta_{x}(A \times B)=\sum_{k=1}^{\infty}\left(1-\delta_{x k}\right) 1_{\left\{\tau_{x}(k) \in A, \sigma_{x}(k) \in B\right\}}
$$

- recall the definitions of age at $k$ th bearing, and type of the child then born; the Ulam-Harris numbering consistently refers to the actual population. Note that the latter is not affected by the value of $\delta_{x}$, so that given $g_{x}, \delta_{x}$ and the actual population are independent. Define

$$
\epsilon_{x}:=\mathbb{E}_{s}\left[\delta_{x} \mid \mathfrak{G}_{x}\right]
$$

and write $\epsilon\left(z_{\tau_{x}}\right)$ for $\epsilon_{x}$ in case of population-size dependence.

We wish to study how dependence mirrors itself in population growth. Therefore, the way of measuring the latter, the characteristic $\chi_{x}$ should not itself be affected by the population history, except through individual type. And indeed natural characteristics are not, like $1_{S \times \mathbb{R}}$, or those counting the number of individuals of certain types, $1_{A \times \mathbb{R}_{+}}, A \in \varphi^{\varphi}$. And the live population, counted by $1_{S \times[0, \lambda]}$, is of this form, provided life span is independent of the population, given type and future reproductions.

We shall call a characteristic conditionally population independent, if given $x$ 's type $\sigma_{x}$ and all the reproductions $\xi_{x^{\prime}}$ of individuals $x^{\prime}$ stemming from $x$, the $\chi_{x}$ 's are i.i.d.

Theorem 1: Let $\left\{z_{t}^{\chi}\right\}$ be the population as counted by a conditionally population independent characteristic $\chi$. Assume that reproductions $\xi$ are finite and stochastically minorized by the reproductions of a general Malthusian branching population with the finite reproduction kernel $\mu$ (defining the Malthusian parameter $\alpha$ stable type distribution $\pi$ and fitness $h$ ). Assume that $w_{t}:=e^{-\alpha t} z_{t}^{\chi}$ is tight.

Further, suppose that for some constant $K$

for all $x \in I$ and $t \geq 0$, and that

$$
\mathbb{E}_{s}\left[z_{t-\tau_{x}}^{\chi}(x) / z_{t}^{\chi} \mid \mathfrak{g}_{x}\right] \leq K / z_{\tau}^{\chi}
$$




$$
\sum_{x} \mathbb{E}_{s}\left[\epsilon_{x} / z_{\tau_{x}}^{\chi}\right]<\infty
$$

Then, $w_{t}$ tends in probability to some finite $w \geq 0$, not identically zero.

We shall refer to the basic inequality

$$
\mathbb{E}_{s}\left[z_{t-\tau_{x}}^{\chi}(x) / z_{t}^{\chi} \mid \mathfrak{g}_{x}\right] \leq K / z_{\tau_{x}}^{\chi}
$$

(for all $t \geq 0$ on the set of non-extinction, or in a suitable interpretation otherwise) as the condition of symmetric growth.

The theorem has one immediate and two less direct corollaries:

Corollary 2: In the population-size dependent case, besides tightness and symmetric growth (and the characteristic being conditionally population independent) assume that $\mathbb{E}_{s}\left[z_{\tau_{X_{n}}}^{\chi}\right]=O(n)$,
and that $\epsilon(t) / t$ is ultimately concave, as $t \rightarrow \infty$. Then

$$
\sum \epsilon(n) / n<\infty
$$

implies that Malthusianess carries over.

Corollary 3: For population-size-dependent Galton-Watson processes with $z_{t}=z_{t}^{\chi}=z_{[t]}$, an $m(n)$ denoting the expected number of individual offspring in a generation of size $n$,

$$
\sum \epsilon(n) / n<\infty \Leftrightarrow \sum\{m(n)-m\} / n<\infty \Rightarrow z_{n} \sim m^{n} w
$$

for a non-zero random variable $w$, provided $\epsilon$ can be interpreted as concave.

For the tumor example, we need a concept of growth fraction: Let $c_{t}$ be the number of cycling cells at time $t$ and $z_{t}$ the total tumor size, i.e., cycling plus quiescent cells. The growth fraction is called strictly positive if $\limsup _{T \rightarrow \infty} \mathbb{E}\left[z_{T} / c_{T}\right]<\infty$, the limsup being over larger and larger stopping times.

Corollary 4: Provided it has a strictly positive growth fraction and the process $w_{t}:=e^{-\alpha t} z_{t}$ is tight, then the cell age and tumor size dependent growth model exhibits balanced exponential growth with the same Malthusian parameter $\alpha$ as the limiting non-tumor-size dependent model, provided only

$$
\sum \epsilon(n) / n<\infty
$$

Proof of the theorem: First some notation: Twiddled processes count only imaginary individuals, $\tilde{z}_{t-\tau_{x}}^{\chi}(x)$ thus standing for the imaginary process starting from the individual $x$ in the actual population at time $\tau_{x}$. Similarly, $\left.\widetilde{w}_{t-\tau_{x}}^{\chi}(x):=e^{-\alpha(t-\tau}\right)_{z_{t-\tau}}^{\chi}(x)$ etc.

For any $u, z_{t}^{u}$ recorded the summed $\chi$-values at time $t+u$ of all individuals born up to $u$ and all individuals not stemming from an aborted individual after $u$. If $\delta_{x u}=0$ precisely if $x$ stems from an $x^{\prime}$ with $\tau_{x^{\prime}}>u$ and $\delta_{x^{\prime}}=1$, and $\delta_{x u}=1$ otherwise, thus

$$
z_{t}^{u}=\sum_{\tau_{x} \leq t+u} \delta_{x u} \chi_{x}\left(t+u-\tau_{x}\right)
$$

In analogy with other notation, $w_{t}^{u}:=e^{-\alpha(t+u)} z_{t}^{u}$.

Clearly,

and

$$
0 \leq z_{t+u}^{\chi}-z_{t}^{u} \leq \sum_{\tau_{x}>u} \delta_{x} z_{t+u-\tau_{x}}^{\chi}(x)
$$

Thus for any $\epsilon^{\prime}, v>0$,

$$
\left|w_{t+u}-w_{t}^{u}\right| \leq w_{t+u} \sum_{\tau}>\delta_{x} \frac{z_{t+u-\tau_{x}}^{\chi}(x)}{z_{t+u}^{\chi}} .
$$




$$
\mathbb{P}_{s}\left(\left|w_{t+u}-w_{t}^{u}\right|>\epsilon^{\prime}, w_{t+u} \leq v\right) \leq\left(v / \epsilon^{\prime}\right) K \mathbb{E}_{s}\left[\sum_{\tau_{x}>u} \delta_{x} / z_{\tau_{x}}^{\chi}\right]
$$

for all $t$ and starting types $s \in S$. But

$$
\left|w_{t+u}-w_{t^{\prime}+u}\right| \leq\left|w_{t+u}-w_{t}^{u}\right|+\left|w_{t}^{u}-w_{t^{\prime}}^{u}\right|+\left|w_{t^{\prime}+u}-w_{t^{\prime}}^{u}\right|
$$

Since

$$
w_{t}^{u}=\sum_{x \in I_{u}} e^{-\alpha \tau} x \widetilde{w}_{t+u-\tau}(x) \rightarrow \sum_{x \in I_{u}} e^{-\alpha \tau} x \widetilde{w}(x),
$$

as $t \rightarrow \infty$, the twiddled daughter processes being independent individual super-critical branching process with Malthusian parameter $\alpha$, it follows that

Hence,

$$
\lim _{t, t^{\prime} \rightarrow \infty} \mathbb{P}_{s}\left(\left|w_{t}^{u}-w_{t^{\prime}}^{u}\right|>\epsilon^{\prime}\right)=0
$$

$$
\operatorname{limsupP}_{t, t^{\prime} \rightarrow \infty}\left(\left|w_{t+u} \wedge v-w_{t^{\prime}+u} \wedge v\right|>\epsilon^{\prime}\right) \leq 2\left(v / \epsilon^{\prime}\right) K \mathbb{E}_{s}\left[\sum_{\tau_{x}>u} \delta_{x} / z_{\tau}^{\chi}\right]
$$

Since $u$ can be chosen as to render the right-hand side arbitrarily small, the convergence in probability follows by completeness and tightness.

Proof of Corollary 3: The characteristic used to count the numbers in different generations is trivially population independent. As $m(n) \epsilon(n)=m(n)-m$ and $1<m \leq m(n) \leq m(1)$, the equivalence of the two conditions is obvious. Symmetric growth follows by - symmetry: Let $x \in N^{k}$ so that $\tau_{x}=k$ and $\mathscr{G}_{x}=\mathscr{B}_{k}$, the $\sigma$-algebra generated by the first $k$ generations. With $t=n$

since

$$
\mathbb{E}\left[z_{t-\tau}^{\chi}(x) / z_{t}^{\chi} \mid \mathfrak{G}_{x}\right]=\mathbb{E}\left[z_{n-k}(x) / z_{n} \mid \mathscr{B}_{k}\right]=1 / z_{k}
$$

$$
z_{n}=\sum_{x \in \mathscr{Z}_{k}} z_{n-k}(z) \text { and }\left|\mathscr{Z}_{k}\right|=z_{k}
$$

if $\mathscr{Z}_{k}$ is the set of realized individuals in the $k$ th generation/

To get hold of $\sum_{n} \mathbb{E}\left[\epsilon\left(z_{\tau_{X_{n}}}\right) / z_{\tau_{X_{n}}}\right]<\infty$, note that

$$
z_{\tau_{X_{n}}}=z_{k} \Leftrightarrow y_{k-1}<n \leq y_{k}
$$

where $y_{k}:=\sum_{j=0^{2}}^{k}$ is the total population up to $k$. Thus,

$$
\sum_{n} \epsilon\left(z_{\tau_{X_{n}}}\right) / z_{\tau_{X_{n}}}=\sum_{k} z_{k} \epsilon\left(z_{k}\right) / z_{k}
$$

and

$$
\begin{gathered}
\sum_{n} \mathbb{E}\left[\epsilon\left(z_{\tau_{X_{n}}}\right) / z_{\tau_{X_{n}}}\right]-\sum_{k} \mathbb{E}\left[\epsilon\left(z_{k}\right)\right] \\
\leq \sum_{k} \mathbb{E}\left[\epsilon\left(z_{k}\right)\right] \leq \sum_{k} \epsilon\left(m(1)^{k}\right),
\end{gathered}
$$

which converges precisely if $\sum_{n} \epsilon(n) / n<\infty$ by an elementary analytic lemma, cf. [11]. The same condition and a similar analytic argument yield $\mathbb{E}[w]<\infty$, and hence tightness (Theorem 5 of $o p$. cit.).

Proof of Corollary 4: In this case, it is the growth of $z_{\tau_{X}}$ that is directly mastered, whereas the symmetric growth inequality causes difficulties. Indeed, ${ }^{\tau} X_{n}$

$$
z_{\tau_{X_{1}}}=1, z_{\tau_{X_{2}}}=z_{\tau_{X_{3}}}=2, z_{\tau_{X_{4}}}=z_{\tau_{X_{5}}}=3, \ldots, z_{\tau_{X_{2 n}}}=z_{\tau_{X_{2 n+1}}}=n+1
$$


on the set where the population does not die out. (Conditional population independence of the characteristic is obvious again.)

For any $x \in I$ with $\tau_{x}<\infty$, define the set

$$
A_{x}:=\left\{x^{\prime} \in I ; \tau_{x^{\prime}} \leq \tau_{x} \text { and a daughter of } x^{\prime} \text { cycles at } \tau_{x}\right\} \cup\{x\} .
$$

By the line $[5,17]$ property of $A_{x}$,

$$
\sum_{x^{\prime} \in A_{x}} z_{t-\tau_{x^{\prime}}}\left(x^{\prime}\right) \leq z_{t}
$$

The inequality comes from the quiescent cells born before those in $A_{x}$.

But the $z_{u}\left(x^{\prime}\right)$ 's do not decrease in $u$ whereas $p(s)$ does not increase in $s$ :

$$
1 \geq \sum_{x^{\prime} \in A_{x}} \mathbb{E}\left[z_{t-\tau_{x^{\prime}}}\left(x^{\prime}\right) / z_{t} \mid \mathfrak{G}_{x}\right] \geq\left|A_{x}\right| \mathbb{E}\left[z_{t-\tau_{x}}(x) / z_{t} \mid \mathfrak{G}_{x}\right] .
$$

But $\left|A_{x}\right|$ must be $\geq c_{\tau_{x}} / 2$. It is clear from the proof of the theorem that now it only remains to prove that

But

$$
\sum_{n} \mathbb{E}\left[\epsilon(n) / c_{\tau_{X_{n}}}\right]<\infty
$$

$$
\mathbb{E}\left[\epsilon(n) / c_{\tau_{X_{n}}}\right] \sim \epsilon(n) / n \mathbb{E}\left[z_{\tau_{X_{n}}} / c_{\tau_{X_{n}}}\right] \sim \epsilon(n) / n
$$

by the assumption of a strictly positive growth factor.

\section{Remarks:}

1. It could well be argued that population size dependence in the form of an albeit imaginary abortion probability should be on $z_{\tau_{x}}$ - rather than on $z_{\tau_{x}}$, so that those born at the very same instant as $x$ do not influence its destiny. This would matter in lattice cases, like the Galton-Watson process. But conceptually, it is a minor variation only.

2. If there is strong symmetric growth, say in the sense that

$$
\mathbb{E}_{s}\left[\sup _{t} z_{t-\tau}^{\chi}(x) / z_{t}^{\chi} \mid \mathfrak{G}_{x}\right] \leq K / z_{\tau}^{\chi}
$$

on the set of non-extinction, a.s. Malthusianess would transfer as well.

\section{References}

[1] Cohn,H. and Klebaner, F., Geometric rate of growth in Markov chains with applications to population-size-dependent models with dependent offspring, Stoch. Anal. Appl. 4 (1986), 283-307.

[2] Gyllenberg, M. and Webb, G.F., A nonlinear structured population model of tumor growth with quiescence, J. Math. Biol. 28 (1990), 671-694.

[3] Gyllenberg, M. and Webb, G.F., Asynchronous exponential growth of semigroups of nonlinear operators, J. Math. Anal. Appl. 167:2 (1992), 443-467.

[4] Jagers, P., Branching Processes with Biological Applications, J. Wiley and Sons, Chichester 1975.

[5] Jagers, P., General branching processes as Markov fields, Stoch. Proc. Appl. $32: 2$ (1989), 183-212.

[6] Jagers, P., Towards dependence in general branching processes, In: Classical and Modern Branching Processes (ed. by K. Athreya and P. Jagers), Springer-Verlag, New York 1997. 
[7] Jagers, P., Coupling and population dependence in branching processes, Dept. of Mathematics, Chalmers University of Technology and Gothenburg University (1996), submitted.

[8] Keller, G., Kersting, G. and Rösler, U., On the asymptotic behavior of discrete time stochastic growth processes, Ann. Prob. 15 (1987), 305-343.

[9] Kersting, G., On recurrence and transience of growth models, J. Appl. Prob. 23 (1986), 614-625.

[10] Klebaner, F., Geometric rate of growth in population-size-dependent branching processes, J. Appl. Prob. 21 (1984), 40-49.

[11] Klebaner, F., A limit theorem for population-size-dependent branching processes, J. Appl. Prob. 22:11 (1985), 48-57.

[12] Klebaner, F., Geometric growth in near-supercritical population size dependent multitype Galton-Watson processes, Ann. Prob. 17:4 (1989), 1466-1477.

[13] Klebaner, F., Asymptotic behavior of Markov population processes with asymptotically linear rate of change, J. Appl. Prob. 31 (1994), 614-625.

[14] Küster, P., Generalized Markov branching processes with state-dependent offspring distribution, Z. Wahr. verw. Geb. 64 (1983), 475-503.

[15] Küster, P., Asymptotic growth of controlled Galton-Watson processes, Ann. Prob. 13:4 (1985), 1157-1178.

[16] Lindvall, T., Lectures on the Coupling Method, Wiley, Chichester 1992.

[17] Neveu, J., Arbres et processus de Galton-Watson, Ann. Inst. H. Poincaré 22 (1986), 199207.

[18] Niemi, S. and Nummelin, E., On non-singular renewal kernels with an application to a semigroup of transition kernels, Stoch. Proc. Appl. 22 (1986), 177-202.

[19] Nummelin, E., General Irreducible Markov Chains and Non-negative Operators, Cambridge University Press, Cambridge 1984.

[20] Olofsson, P., General Branching Processes with Local Dependencies, Thesis, Dept. of Math., Chalmers and Gothenburg Univ. 1994.

[21] Olofsson, P., Branching processes with local dependencies, Ann. Appl. Prob. (1996), to appear.

[22] Rittgen, W., Über das qualitative Verhalten populationsabhängiger Markoffscher Verzweigungsproxesse, Fachbereich Mathematik der Johannes-Gutenberg-Universität, Mainz 1986.

[23] Sevast'janov, B.A. and Zubkov, A.M., Controlled branching processes, Teor. Veroyat. Primen. 19 (1974), 15-25.

[24] Shurenkov, V.M., Ergodičeskie Processy Markova (Ergodic Markov Processes), Nauka, Moscow 1989. 


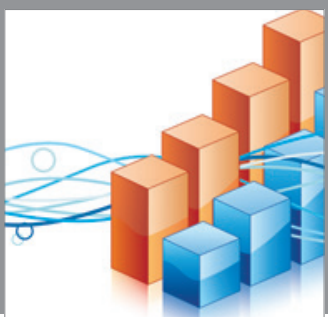

Advances in

Operations Research

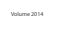

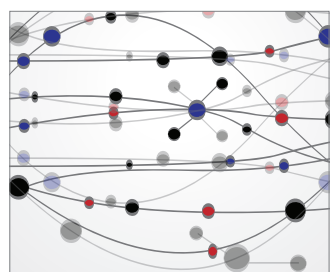

\section{The Scientific} World Journal
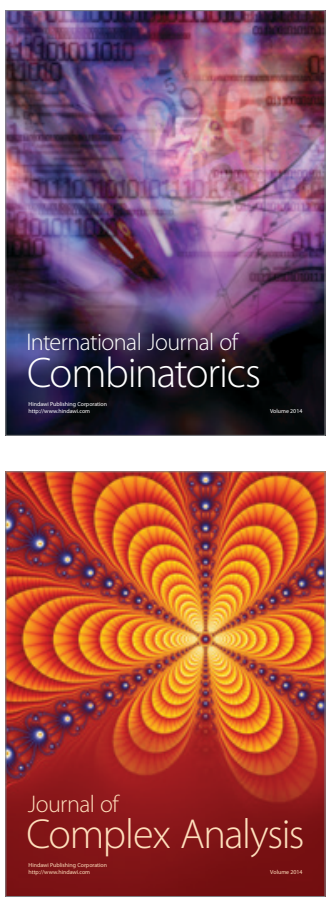

International Journal of

Mathematics and

Mathematical

Sciences
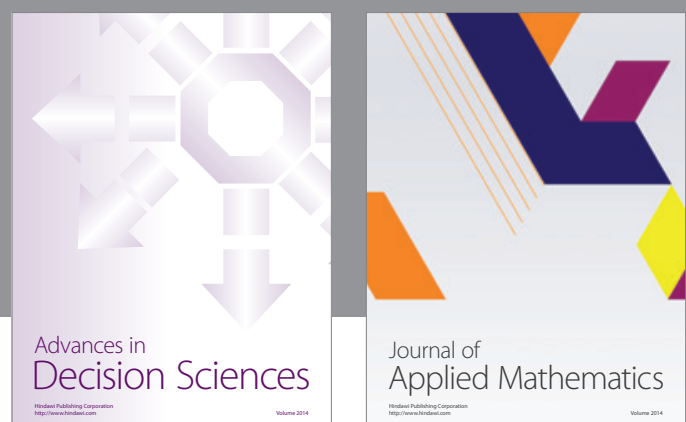

Journal of

Applied Mathematics
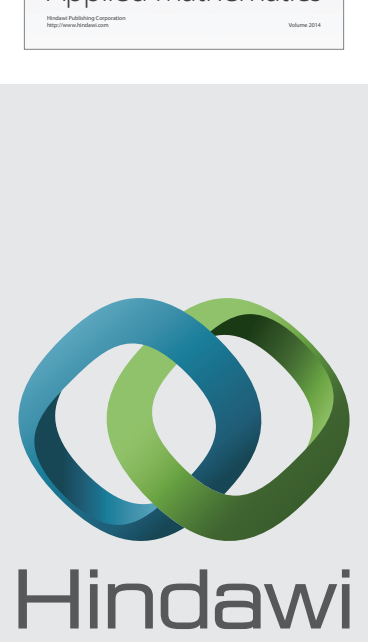

Submit your manuscripts at http://www.hindawi.com
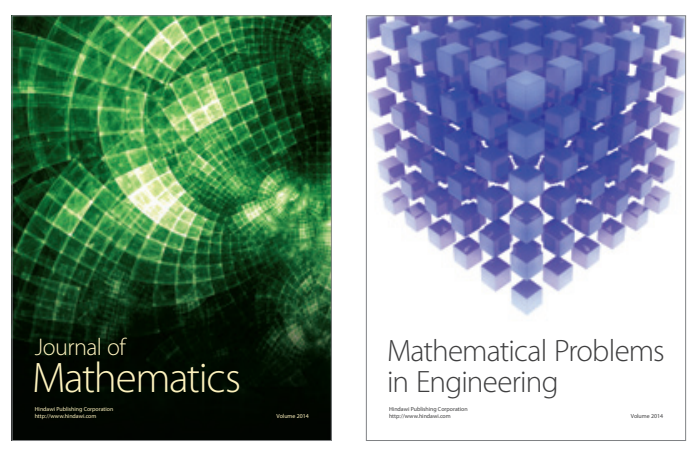

Mathematical Problems in Engineering
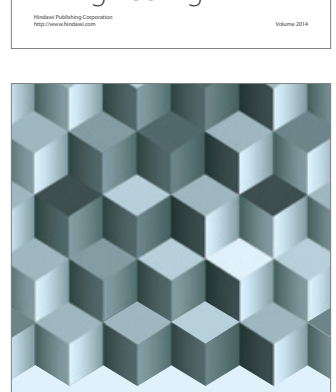

Journal of

Function Spaces
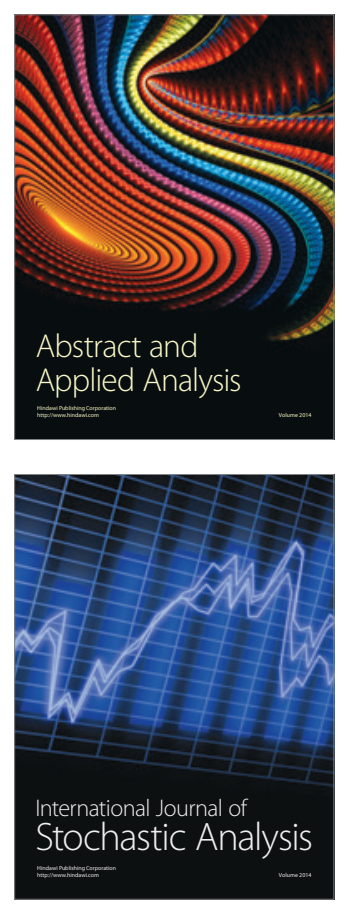

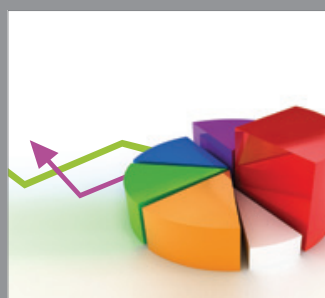

ournal of

Probability and Statistics

Promensencen
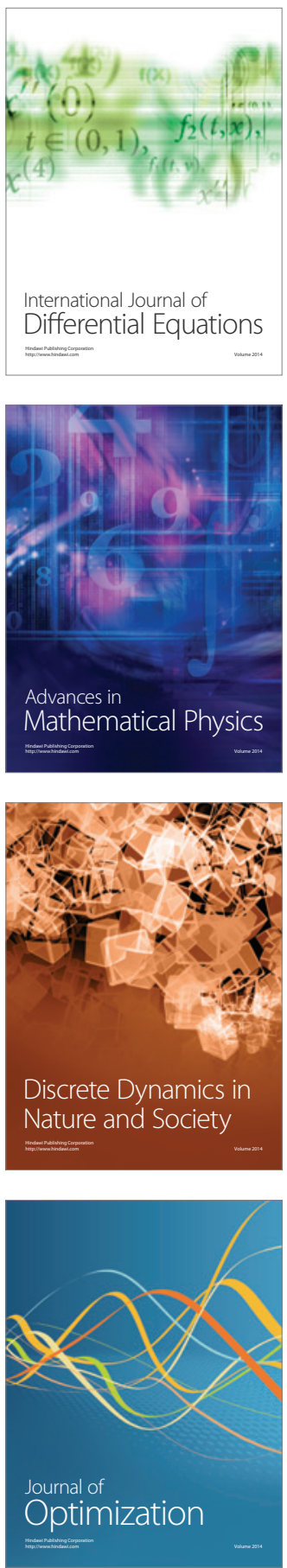